\title{
Study of nuclear structure influencing fusion reactions
}

\author{
J. Khuyagbaatar ${ }^{1}$, K. Nishio ${ }^{2}$, D. Ackermann ${ }^{1}$, M. Block ${ }^{1}$, S. Heinz ${ }^{1}$, F.P. Heßberger ${ }^{1}$, S. Hofmann ${ }^{1}$, H. Ikezoe ${ }^{2}$, \\ B. Kindler ${ }^{1}$, B. Lommel ${ }^{1}$, H. Makii ${ }^{2}$, S. Mitsuoka ${ }^{2}$, I. Nishinaka ${ }^{2}$, Y. Wakabayashi ${ }^{2}$, and S. Yan ${ }^{1,3}$ \\ ${ }^{1}$ GSI Helmholzzentrum für Schwerionenforschung GmbH, 64291 Darmstadt, Germany \\ ${ }^{2}$ Japan Atomic Energy Agency, 319-1184 Tokai, Ibaraki, Japan \\ ${ }^{3}$ China Institute of Atomic Energy, P.O. Box 275 (10), Beijing, China
}

\begin{abstract}
The influence of the nuclear structure of projectile and target nuclei on the capture cross-sections was investigated in the fusion reactions ${ }^{34} \mathrm{~S}+{ }^{204,206,208} \mathrm{~Pb}$ and ${ }^{36} \mathrm{~S}+{ }^{204,206,208} \mathrm{~Pb}$. Capture cross-sections were deduced from the fission fragments measured with multi-wire proportional counters. Significantly enhanced capture-fission cross-sections were observed for the ${ }^{34} \mathrm{~S}$ induced reactions compared to ${ }^{36} \mathrm{~S}$ at beam energies below the interaction barriers. The experimental results were compared to a coupled-channel calculation. The enhancement was explained by strong couplings to vibrational states of the ${ }^{34} \mathrm{~S}$ with ${ }^{204,206,208} \mathrm{~Pb}$. The capture and evaporation residue cross-sections of ${ }^{34} \mathrm{~S}+{ }^{208} \mathrm{~Pb}$ and ${ }^{36} \mathrm{~S}+{ }^{206} \mathrm{~Pb}$ reactions which lead to the same compound nucleus ${ }^{242} \mathrm{Cf}$ are compared and discussed.
\end{abstract}

\section{INTRODUCTION}

The most successful type of reaction for the production of heavy and superheavy nuclei (SHN) is fusion of two colliding nuclei to form an excited compound nucleus which in turn de-excites by evaporation of few neutrons $[1,2]$. However, cross-sections for the production of heavy and superheavy nuclei in fusion reactions decrease rapidly with increasing number of protons in the compound nucleus. Thus, the possibilities to search for SHN and to investigate the nuclear structure of these nuclei are limited by their production rates [1]. Therefore, the choice of the reaction which gives highest fusionevaporation cross-sections is an important issue. The cross-section values of the 2 n-evaporation channels from fusion reactions ${ }^{36} \mathrm{~S}+{ }^{206} \mathrm{~Pb}$ and ${ }^{34} \mathrm{~S}+{ }^{208} \mathrm{~Pb}$ leading to the same compound nucleus ${ }^{242} \mathrm{Cf}$ have been measured in [3] and they differs by factor of five. This difference has been attributed to larger negative Q-value of the ${ }^{36} \mathrm{~S}$ (semi-magic) induced reaction.

Recently, these cross-sections have been re-measured exploiting improved experimental technique $[4,5]$. The results showed an even larger difference than the reported in [3].

The evaporation residue (ER) cross-sections depends on capture cross-sections, which is largely influenced by a reaction $\mathrm{Q}$-value and coupling to collective states of colliding nuclei in the sub-barrier energy region [6].

We aimed to measure the cross-sections of the reactions of ${ }^{34} \mathrm{~S}$ and ${ }^{36} \mathrm{~S}$ projectiles with various lead targets by detecting the fission fragments in order to understand the unexpectedly large difference of ER cross-sections between the ${ }^{36} \mathrm{~S}+{ }^{206} \mathrm{~Pb}$ and ${ }^{34} \mathrm{~S}+{ }^{208} \mathrm{~Pb}$. Moreover, these combinations at absent ground-state deformation of projectile and target nuclei are suitable for the study of the influence of the nuclear structure degrees of freedom of ${ }^{34} \mathrm{~S}$ and ${ }^{36} \mathrm{~S}$ nuclei on the capture process.

\section{EXPERIMENTAL SETUP}

The experiment was performed at the JAEA-tandem accelerator in Tokai, Japan. The sulfur beams $\left({ }^{34} \mathrm{~S}\right.$ and ${ }^{36} \mathrm{~S}$ ) were extracted from the negative ions source and were accelerated up to energies of $E_{\text {lab }}=(160-200) \mathrm{MeV}$ with intensities from 0.1 to $3.0 \mathrm{pnA}$. Metallic lead targets with thicknesses of $100 \mu \mathrm{g} / \mathrm{cm}^{2}$ were prepared at the target laboratory of GSI, Germany. They were made by evaporating isotopically enriched $(>99.9){ }^{204,206,208} \mathrm{~Pb}$ on carbon foils with an average thickness of $40 \mu \mathrm{g} / \mathrm{cm}^{2}$. The opposite side of the targets was covered by the thin 10 $\mu \mathrm{g} / \mathrm{cm}^{2}$ carbon layer. Targets were mounted at an angle of $45^{\circ}$ to the beam axis by the thin carbon layer in the beam direction. The present experimental setup and analysis of measured data are similar to described in [7]. Two position-sensitive multi-wire proportional counters (MWPCs) were used to detect both fission fragments in coincidence. Fission fragments emission angles $\theta_{i}$ and out-of-plane angles $\varphi_{i}$ were defined as shown in Fig. 1. The MWPC consisted of a gold coated Mylar foil (cathode), sandwiched by two grounded wire planes. The wires with $2 \mathrm{~mm}$ pitch covered an area of $200 \mathrm{~mm}$ and $120 \mathrm{~mm}$ in horizontal and vertical direction, respectively. 

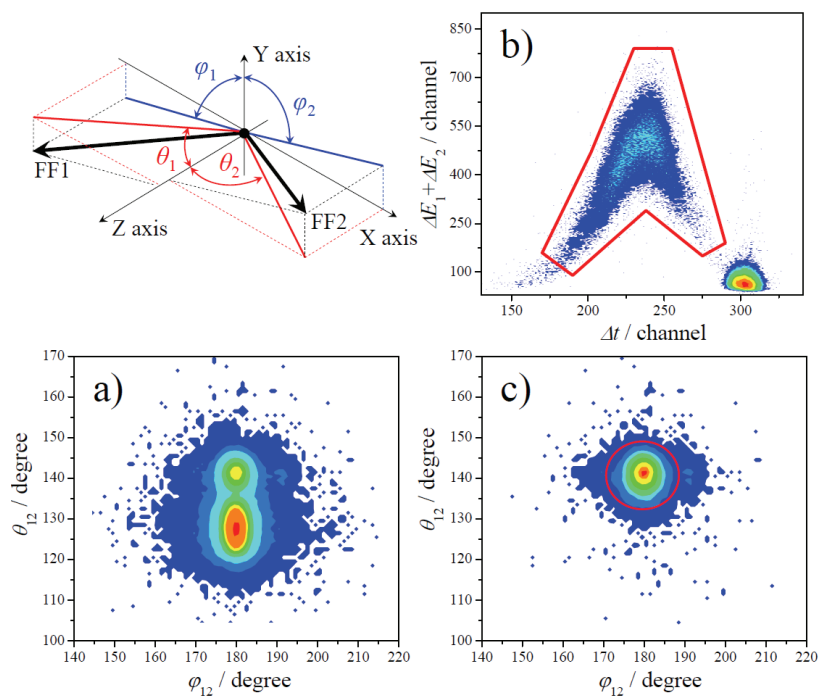

Fig. 1. (Color Online). Upper left: Definition of the emission angles $\theta_{1}$ and $\theta_{2}$ and of the out-of-plane angles $\varphi_{1}$ and $\varphi_{2}$. a) Events plotted on the $\varphi_{12}$ vs $\theta_{12}$ plane. b) Plot on the $\Delta t$ and $\left(\Delta E_{1}+\Delta E_{2}\right)$ plane. c) Same as a), but obtained with a gate on the events as shown in $b$ ).

The entrance windows of both detectors were Mylar foils of $3 \mu \mathrm{m}$ thickness. Detectors were operated with isobutane gas at a pressure of 3 Torr. Detectors were located on side of the target at a distance of $211 \mathrm{~mm}$. The MWPC1 was located at the angle $\theta_{1}=-57.0^{\circ}$ and covered a range of $-86.0^{\circ} \leq \theta_{1} \leq-36.0^{\circ}$. MWPC 2 was located at the angle $\theta_{2}=+90.0^{\circ}$ and covered a range of $65.0^{\circ} \leq \theta_{2} \leq 108.0^{\circ}$. For the out-of-plane angle, the MWPC1 and MWPC2 covered a range of $72.0^{\circ} \leq \varphi_{1} \leq 108.0^{\circ}$ at $\theta_{1}=-57.0^{\circ}$ and $74.1^{\circ} \leq \varphi_{2} \leq 105.9^{\circ}$ at $\theta_{2}=+90.0^{\circ}$. Folding angle $\theta_{12}$ and outof-plane $\varphi_{12}$ angles were defined as $\theta_{1}+\theta_{2}$, and as $\varphi_{2}-\varphi_{1}$, respectively. The time difference $\Delta t$ between the two fragments was measured with start and stop signals obtained from MWPC2 and MWPC1, respectively. Induced charges in both MWPCs contain information on the energy deposition $\Delta E_{1}$ and $\Delta E_{2}$ of particles passing through the detectors. An example of the analysis of fission fragments from the ${ }^{34} \mathrm{~S}+{ }^{208} \mathrm{~Pb}$ reaction is shown in Fig. 1. These data were obtained at the center-of-mass energy $E_{c . m .}=158.7 \mathrm{MeV}$ (in the middle of the target). Observed events are shown in Fig. 1a on the $\left(\theta_{12}, \varphi_{12}\right)$ plane. Clearly two centroides corresponding to fission and elastic scattering are visible. A two dimensional contour plot of the detected events on the $\left(\Delta E_{1}+\Delta E_{2}, \Delta t\right)$ plane (Fig. 1b) provides an additional information which can be used to select only fission events. Events are marked in Fig. 1b (selected by an arrowhead curve) belong to fission fragments. Fig. 1c also shows events on the $\left(\theta_{12}, \varphi_{12}\right)$ plane, but for events located within the gate marked in Fig. 1b. We define the fission events as those recorded within the gate marked in Fig. $1 \mathrm{~b}$.

The accumulated beam dose was deduced detecting elastically scattered particles at an angle of $26.5^{\circ}$ relative to the beam axis and with a solid angle of $1.96 \mathrm{msr}$.

\section{EXPERIMENTAL RESULTS}

Experimental fission cross-sections are shown in Fig. 2 as a function of $E_{c . m} / V_{i n t}$, where $E_{c . m .}$-is the center-ofmass energy and $V_{\text {int }}$-is the interaction barrier height (see below). The cross-section was deduced from the angular distribution $d \sigma_{\text {fiss }} / d \Omega$ in the range of $85^{\circ}<\theta_{12}<125^{\circ}$. Angular distributions of fissions were fitted by the function $d W(\theta) / d \Omega$ given in [8], which was then integrated over the solid angle to yield the total fission cross-sections. Systematical uncertainty of $35 \%$ was estimated for the deduced cross-section values due to the limited angular range coverage of our experimental setup [7].

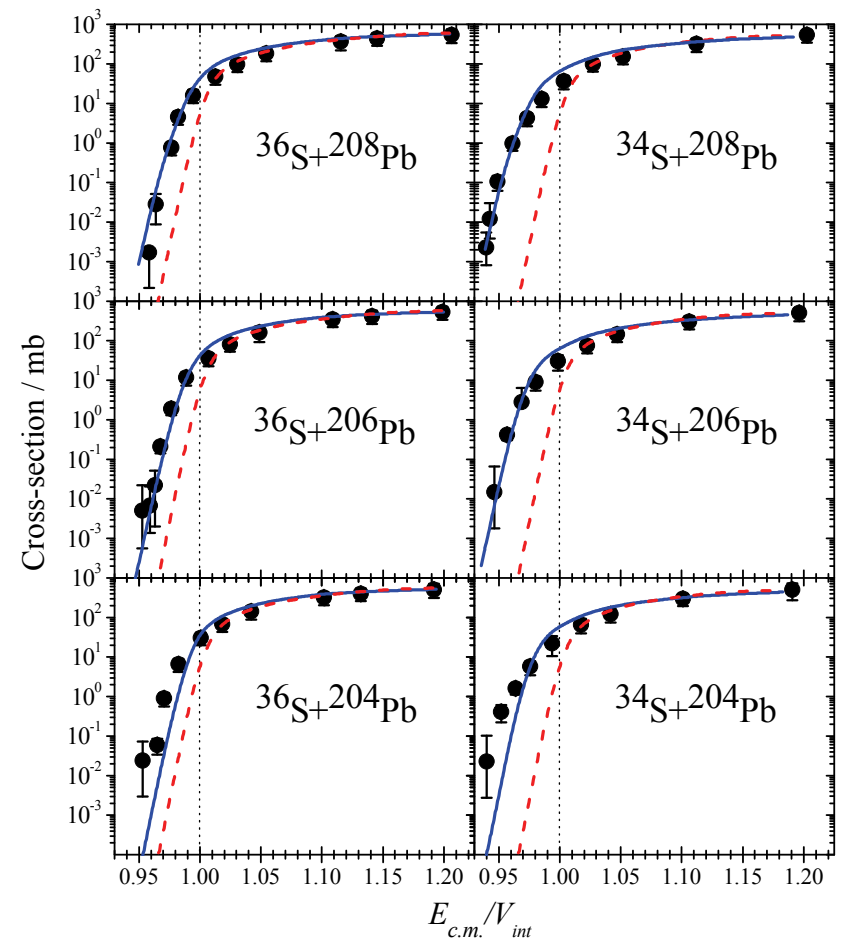

Fig. 2. Measured fission cross-sections (solid circles) plotted as a function of the ratio of center-of-mass energy and interaction barrier height. Dashed and solid curves represent the CC calculations without coupling and with coupling, respectively. Vertical dotted lines indicate the interaction barrier position.

The behaviour of fission cross-sections as a function of beam energies is similar for ${ }^{36} \mathrm{~S}+{ }^{204,206,208} \mathrm{~Pb}$ reactions and they decrease rapidly at low beam energies (same for ${ }^{34} \mathrm{~S}$ ). This indicates that the change in the nuclear structure of the target nuclei ${ }^{204,206,208} \mathrm{~Pb}$ has a small influence to the entrance channel. However, the slopes of the excitation functions in the sub-barrier energy region are different between ${ }^{36} \mathrm{~S}$ and ${ }^{34} \mathrm{~S}$.

In order to discuss the influence of nuclear structure properties on the capture cross-section, we performed coupled-channel (CC) calculations. In our CC calculations we used the computer code CCFULL [9]. Uncoupled (Wood-Saxon) potential parameters were established using the literature data from [10]. The same potential depth $200 \mathrm{MeV}$ was used for all reactions according to [10]. Radii and diffuseness parameters were chosen in such a way that the fission cross-sections above the interaction barriers are reproduced. Parameters for 
nuclear reactions of projectile and target combinations are given in Table 1.

Table 1. Scaling parameters, reaction Q-value, interaction barriers used in the present $\mathrm{CC}$ calculations $\left(V_{\text {int }}(C C)\right)$ and according to the Bass prediction $\left(V_{\text {int }}(\right.$ Bass $\left.)\right)$ [11] for ${ }^{34,36} \mathrm{~S}+{ }^{204,206,208} \mathrm{~Pb}$ reactions.

\begin{tabular}{|c|c|c|c|c|}
\hline Reaction & $\begin{array}{c}\text { Scaling } \\
\text { parameter }\end{array}$ & $\begin{array}{c}\text { Q-value } \\
/ \mathrm{MeV}\end{array}$ & $\begin{array}{c}V_{\text {int }}(C C) \\
/ \mathrm{MeV}\end{array}$ & $\begin{array}{c}V_{\text {int }} \text { (Bass) } \\
/ \mathrm{MeV}\end{array}$ \\
\hline${ }^{36} \mathrm{~S}+{ }^{208} \mathrm{~Pb}$ & 142.19 & -113.9 & 141.1 & 144.8 \\
\hline${ }^{36} \mathrm{~S}+{ }^{206} \mathrm{~Pb}$ & 142.49 & -113.8 & 141.7 & 145.1 \\
\hline${ }^{36} \mathrm{~S}+{ }^{204} \mathrm{~Pb}$ & 142.78 & -113.8 & 142.4 & 145.4 \\
\hline${ }^{34} \mathrm{~S}+{ }^{208} \mathrm{~Pb}$ & 143.16 & -111.0 & 142.7 & 145.7 \\
\hline${ }^{34} \mathrm{~S}+{ }^{206} \mathrm{~Pb}$ & 143.46 & -111.7 & 143.2 & 146.0 \\
\hline${ }^{34} \mathrm{~S}+{ }^{204} \mathrm{~Pb}$ & 143.76 & -112.4 & 143.7 & 146.3 \\
\hline
\end{tabular}

The interaction barriers, radii and diffuseness parameters of ${ }^{34} \mathrm{~S}+{ }^{206} \mathrm{~Pb}$ and ${ }^{36} \mathrm{~S}+{ }^{204} \mathrm{~Pb}$ reactions were taken from [10]. Interaction barriers of other reactions were estimated using a fitted linear function of barriers depend on the scaling parameters $Z_{1} \cdot Z_{2} /\left(A_{1}{ }^{1 / 3}+A_{2}{ }^{1 / 3}\right)$ for data obtained in [10]. These values deviate from the Bass predictions [11]. Radii and diffuseness parameters of these reactions were chosen similar to values obtained in [10] and at the same time these values reproduce estimated interaction barriers. The radii and diffuseness parameters from [10] differ from the realistic values. However, the present version of the $\mathrm{CC}$ calculation with such parameters well describes the sub-barrier enhancement. Presently improved versions of CC calculations which describe well both sub-barrier and above-barrier capture cross-sections, using a realistic nuclear potential parameters are available (see in [12] and references therein). Results of calculations are shown in Fig. 2. Dashed curves represent calculations without couplings of the vibratinal states the projectile and target nuclei. Experimental cross-sections at center-of-mass energies above the estimated interaction barriers are well described. They underestimate the experimental crosssections at sub-barrier energies for all reactions. Crosssections down to the lowest measured energies are well described by $\mathrm{CC}$ calculations where the couplings of the lowest lying $2^{+}$and $3^{-}$states in projectile and target nuclei, respectively, were taken into account. Excitation energies (deformation parameters) of $2.127 \mathrm{MeV}(0.252)$ and $3.291 \mathrm{MeV}(0.164)$ were used for $2^{+}$states of ${ }^{34} \mathrm{~S}$ and ${ }^{36} \mathrm{~S}$ nuclei, respectively, according to [13]. Data for 3 states of the $\mathrm{Pb}$ nuclei were taken from [14] and corresponding values are $2.618 \mathrm{MeV}(0.092), 2.648 \mathrm{MeV}$ $(0.108)$ and $2.615 \mathrm{MeV}(0.150)$ for ${ }^{204} \mathrm{~Pb},{ }^{206} \mathrm{~Pb}$ and ${ }^{208} \mathrm{~Pb}$ nuclei, respectively.

Our calculations well describe the experimental results except the reactions involving ${ }^{204} \mathrm{~Pb}$ where the experimental cross-sections are larger than the calculations in both reactions ${ }^{34} \mathrm{~S}+{ }^{204} \mathrm{~Pb}$ and ${ }^{36} \mathrm{~S}+{ }^{204} \mathrm{~Pb}$. This could be related to the structure of the ${ }^{204} \mathrm{~Pb}$ nucleus which has four vacant neutron holes.

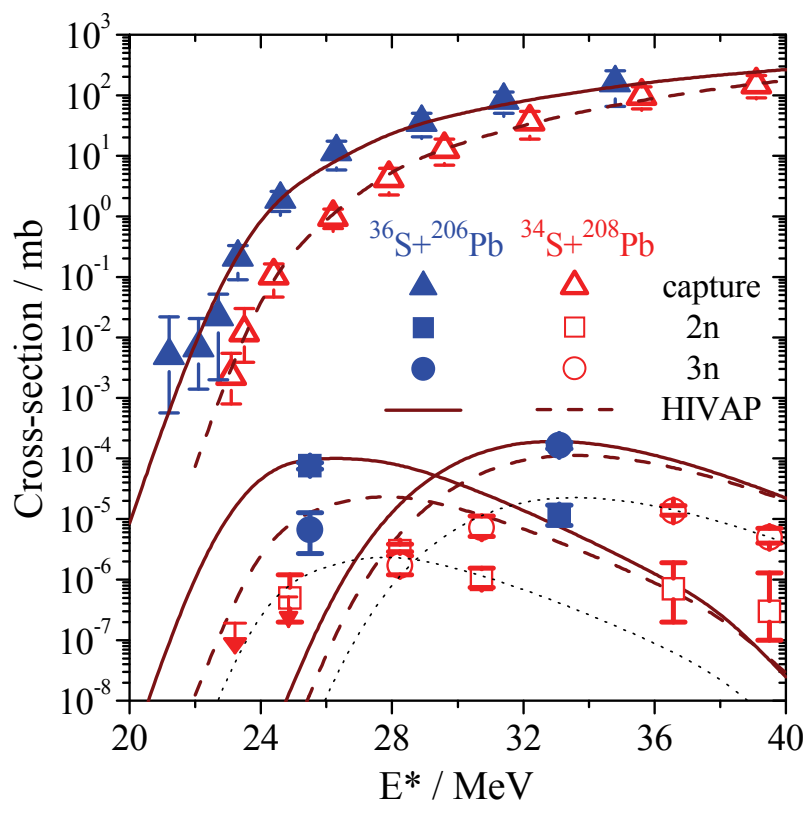

Fig. 3. Experimental data are shown by symbols for the fusion reaction ${ }^{34} \mathrm{~S}+{ }^{208} \mathrm{~Pb}$ and ${ }^{36} \mathrm{~S}+{ }^{206} \mathrm{~Pb}$ both leading to the same compound nucleus ${ }^{242} \mathrm{Cf}$. Lines are the results from HIVAP calculations. Downward arrows mark the upper limits for the cross-section. Dotted lines show the eye-guide for the ${ }^{34} \mathrm{~S}$ evaporation residue data.

The comparison of capture cross-sections of ${ }^{36} \mathrm{~S}+{ }^{206} \mathrm{~Pb}$ and ${ }^{34} \mathrm{~S}+{ }^{208} \mathrm{~Pb}$ reactions shows a significant enhancement due to the entrance channel in case of ${ }^{34} \mathrm{~S}$ (Fig. 2). These values are presented in Fig. 3 as a function of excitation energies of the compound nucleus $\left({ }^{242} \mathrm{Cf}\right)$ together with measured fusion-evaporation cross-sections for $2 \mathrm{n}$ and $3 \mathrm{n}$ channels $[4,5]$. Capture and fusion-evaporation crosssections calculated by the HIVAP [15] code are also shown in Fig. 3. The HIVAP parameters were modified to reproduce the experimental fission cross-sections of both reactions and fusion-evaporation cross-sections for the ${ }^{36} \mathrm{~S}+{ }^{206} \mathrm{~Pb}$ reaction.

About 25 and 10 times higher cross-section values for the $2 \mathrm{n}$ and $3 \mathrm{n}$ evaporation channels (close to the maxima of excitation functions), respectively, were observed in the case of the ${ }^{36} \mathrm{~S}+{ }^{206} \mathrm{~Pb}$ as compared to ${ }^{34} \mathrm{~S}+{ }^{208} \mathrm{~Pb}$. Calculated ER cross-sections overestimate the experimental values for the ${ }^{34} \mathrm{~S}+{ }^{208} \mathrm{~Pb}$ reaction. This discrepancy implies an additional effect which hinders fusion in the ${ }^{34} \mathrm{~S}$ induced reaction. However, it is still a very surprising result that the fusion reactions ${ }^{36} \mathrm{~S}+{ }^{206} \mathrm{~Pb}$ and ${ }^{34} \mathrm{~S}+{ }^{208} \mathrm{~Pb}$ differ so strongly which cannot be explained with the current knowledge. Therefore, further investigations to obtain more experimental data points for ER cross-sections are needed to reveal the excitation functions clearly.

\section{CONCLUSIONS}

The fission cross-sections of the ${ }^{34} \mathrm{~S}$ and ${ }^{36} \mathrm{~S}$ induced reactions on ${ }^{204,206,208} \mathrm{~Pb}$ targets were measured. The excitation functions smoothly change for the different lead isotopes. A larger enhancement of the capture crosssections in the energy region below the interaction barriers were observed in the ${ }^{34} \mathrm{~S}$ induced reactions as 
compared to ${ }^{36} \mathrm{~S}$. The calculations which take into account the couplings to the low lying $2^{+}$and $3^{-}$states in projectile and target nuclei were performed. The calculations describe the experimental results well except for the reaction using ${ }^{204} \mathrm{~Pb} \quad(\mathrm{~N}=122)$ where the experimental fission cross-sections indicates some enhancement. This could be due to the higher order of couplings which involves more states or due to neutron transfer channels. The estimated interaction barriers which well describe the experimental results were significantly lower than the Bass predictions.

Comparison of fission cross-sections of ${ }^{34} \mathrm{~S}+{ }^{208} \mathrm{~Pb}$ and ${ }^{36} \mathrm{~S}+{ }^{206} \mathrm{~Pb}$ reactions show a significant enhancement below the interaction barrier in the case of ${ }^{34} \mathrm{~S}$ due to its nuclear structure. However, ER cross-sections show a significant hindrance in fusion in the case of ${ }^{34} \mathrm{~S}$ which cannot be explained by the reaction Q-value.

\section{ACKNOWLEDGEMENTS}

We thank the JAEA-tandem facility staff for the beam operation. One of us (J.K) wants to thank JAEA for the foreign young scientist's grant which allowed a long term visit at JAEA during which this experiment was performed. Special thanks are due to Dr. V.V Sargsyan, Dr. G.G. Adamian and Dr. N.V. Antonenko of JINR in Dubna for fruitful discussions. This work was supported by a Grant-in-Aid for Scientific Research of the Japan Society for the Promotion of Science.

\section{References}

1. S. Hofmann. and G. Münzenberg, Rev. Mod. Phys. 72, 733 (2000).

2. Yu.Ts. Oganessian, J. Phys. G: Nucl. Part. Phys. 34, R165 (2008).

3. Yu.A. Lazarev et al., Nucl. Phys. A 588, 501 (1995).

4. J. Khuyagbaatar et al., Eur. Phys. J. A 46, 59 (2010).

5. J. Khuyagbaatar et al., to be published.

6. M. Beckerman, Rep. Prog. Phys. 51, 1047 (1988).

7. K. Nishio et al., Phys. Rev. C. 77, 064607 (2008).

8. R. Vandenbosch and J.R. Huizenga, Nuclear Fisson (Academic Press, New York, 1973).

9. K. Hagino, N. Rowley and A.T. Kruppa, Computer Phys. Comm. 123, 143 (1999).

10. D.J. Hinde et al., Phys. Rev. C. 75, 054603 (2007).

11. R. Bass, Phys. Rev. Lett. 39, 265 (1977).

12. T. Ichikawa et al., Phys. Rev. Lett. 103, 202701 (2009).

13. S. Raman et al., Atomic Data and Nuclear data tables. 36, 1 (1987).

14. R.H. Spear, Atomic Data and Nuclear data tables. 42, 55 (1987).

15. W. Reisdorf, Z. Phys. A 300, 227 (1981). 